\title{
NSD Total Choosability of Planar Graphs with Girth at Least Four
}

\author{
Xue Han, Jihui Wang ${ }^{*}$ and Baojian Qiu \\ School of Mathematical Science, University of Jinan \\ Jinan, Shandong, P.R. China, 250022 \\ ${ }^{*}$ Corresponding author
}

\begin{abstract}
A proper total $k$-coloring $\phi$ of a graph $G$ is a mapping from $V(G) \cup E(G)$ to $\{1, \cdots, k\}$ such that no adjacent or incident elements in $V(G) \cup E(G)$ receive the same color. Let $\Sigma_{\phi}(u)$ denote the sum of the colors on the edges incident with the vertex $u$ and the color on $u$. A proper total $k$-coloring of $G$ is called neighbor sum distinguishing if $\Sigma_{\phi}(u) \neq \Sigma_{\phi}(v)$ for each edge $u v \in E(G)$. Let $L_{z}(z \in V(G) \cup E(G))$ be a set of lists of integer numbers, each of size $k$. The smallest $k$ for which for any specified collection of such lists, there exists a neighbor sum distinguishing total coloring using colors from $L_{z}$ for each $z \in V(G) \cup E(G)$ is called the neighbor sum distinguishing total choosability of $G$, and denoted by $c h_{\Sigma}^{T}(G)$. In this paper, we prove that $c h_{\Sigma}^{T}(G) \leq \Delta(G)+3$ for planar graphs with girth at least 4. This implies that Pilsniak and Wozniak' conjecture is true for any planar graphs with girth at least 4 and $\Delta(G) \geq 7$.
\end{abstract} graph

Keywords-NSD total coloring; choosability; girth; planar

\section{INTRODUCTION}

The terminology and notation used but undefined in this paper can be found in [3]. Graphs considered in this paper are finite, simple and undirected. Let $G=(V, E)$ be a graph. We use $V(G), E(G), \Delta(G)$ and $\delta(G)$ to denote the vertex set, edge set, maximum degree and minimum degree of $G$, respectively. Let $d_{G}(v)$ or simply $d(v)$ denote the degree of a vertex $v$ in $G$. A vertex $v$ is called an $l$-vertex if $d(v)=l$, similarly, an $l^{+}$-vertex or an $l^{-}$-vertex if $d(v) \geq l$ or $d(v) \leq l$. Let $d_{i}(v)\left(d_{i^{+}}(v), d_{i^{-}}(v)\right)$ be the number of neighbors of $\mathrm{v}$ with degree $i$ (at least $i$, at most $i$ ) in G. A $k$-face is a face of degree $k$.

Given a graph $G=(V, E)$ and a positive integer $k$, a total $k$-coloring of $G$ is a proper coloring $\phi: V(G) \cup E(G) \rightarrow\{1, \cdots, k\}$, where a proper coloring means every pair of adjacent or incident elements receive different numbers. Given a total $k$-coloring $\phi$ of $G$, let $C_{\phi}(u)$ denote the set of colors of the edges incident to $v$ and the color of $v$. A total $k$-coloring is called adjacent vertex distinguishing if for each edge $u v, C_{\phi}(u)$ is different from $C_{\phi}(v)$. A smallest such $k$ is called the adjacent vertex distinguishing total chromatic number of $G$, denoted by $\chi_{a}^{T}(G)$. Zhang et al. [8] put forward the following conjecture.

Conjecture $1.1^{[8]}$ For any graph $G$ with at least two vertices, $\chi_{a}^{T}(G) \leq \Delta(G)+3$.

Conjecture 1.1 has been proved for a few special cases, such as subcubic graphs, $K_{4}$-minor free graphs and some special planar graphs, see [2,6,7]. Recently, colorings and labellings related to sums of the colors have been studied widely, see the survey paper [1]. In a total $k$-coloring of $G$, let $\Sigma_{\phi}(v)$ denote the sum of colors of the edges incident to $v$ and the color of $v$. If for each edge $u v \in E(G)$, we have $\Sigma_{\phi}(u) \neq \Sigma_{\phi}(v)$, we call such total $k$-coloring a $k$ neighbor sum distinguishing total coloring. The smallest number $k$ is called the neighbor sum distinguishing total chromatic number of $G$, denoted by $\chi_{\Sigma}^{T}(G)$. For neighbor sum distinguishing total colorings, we give the following conjecture due to Pilsniak and Wozniak ${ }^{[5]}$.

Conjecture $1.2^{[5]}$ For any graph $G$ with at least two vertices, $\chi_{\Sigma}^{T}(G) \leq \Delta(G)+3$.

Conjecture 1.2 implies Conjecture 1.1, since it is easy to check that $\chi_{a}^{T}(G) \leq \chi_{\Sigma}^{T}(G)$. Pilsniak and Wozniak ${ }^{[5]}$ proved that Conjecture 1.2 holds for complete graphs, cycles, bipartite graphs and subcubic graphs. For a given graph $G$, let $L_{z}(z \in V(G) \cup E(G))$ be any set of list of integer numbers, each of size $k$. If for any specified collection of such lists, there exists a neighbor sum distinguishing total coloring of $G$ using colors from $L_{z}$ for each $z \in V(G) \cup E(G)$, 
we call such coloring a $k$-neighbor sum distinguishing list total coloring, the smallest $k$ is called the neighbor sum distinguishing total choosability of $G$, and denoted by $c h_{\Sigma}^{T}(G)$. In this paper, we studied the neighbor sum distinguishing total choosability of planar graphs with girth at least 4 and proved the following result.

Theorem 1.1 If $G$ is a planar graph with girth at least 4 and $\Delta(G) \geq 7$, then $c h_{\Sigma}^{T}(G) \leq \Delta(G)+3$.

Clearly, $\chi_{\Sigma}^{T}(G) \leq c h_{\Sigma}^{T}(G)$, so the result above holds also for $\chi_{\Sigma}^{T}(G)$. This implies that Pilsniak and Wozniak' conjecture is true for planar graphs with girth at least 4 and $\Delta(G) \geq 7$. Our approach is based on the discharging method and some other tricks, which have been widely used in coloring theory.

\section{Proof OF TheOrem 1.1}

In order to prove the main result, we need next lemma.

Lemma $2.1^{[4]}$ Suppose $m$ is a positive integer, $L_{j}$ is a set of integers with $\left|L_{j}\right|=l_{j} \geq m$ for each $j \in\{1, \cdots, m\}$, let $T_{m}\left(L_{1}, \cdots, L_{m}\right)=$

$$
\left\{\sum_{i=1}^{m} x_{i} \mid x_{i} \in L_{i}, i \neq j \Rightarrow x_{i} \neq x_{j}\right\} \text {. }
$$

Then

$$
\left|T_{m}\left(L_{1}, \cdots, L_{m}\right)\right| \geq \sum_{j=1}^{m} l_{j}-m^{2}+1 \text {. }
$$

Let $L_{z}(z \in V(G) \cup E(G))$ be any given set of lists of integer numbers, each of size $k$, where $k=\Delta(G)+3$. For simplicity, we use " $k$-nsd list total coloring" to denote " $k$-neighbor sum distinguishing list total coloring". Let $\phi$ be a $k$-nsd list total coloring of planar graph $G$ without adjacent triangles with $\Delta(G) \geq 7$. Assume that $u \in V(G)$ with $d(u) \leq 3$, it is easy to see that $u$ has at most 3 adjacent vertices and 3 incident edges, and the sum obtained at $u$ must be distinct from 3 sums at the adjacent vertices of $u$. So $u$ has at most 9 forbidden colors. Since $\left|L_{u}\right|=k \geq 10$, we may first erase the color of $u$ and recolor it finally. In other words, we may omit the recoloring for all $3^{-}$-vertices in the following discussion.

Our proof proceeds by reduction and absurdum. Assume that $G$ is a counterexample to Theorem 1.1 such that $|V(G)|+|E(G)|$ is as small as possible. Obviously, $G$ is connected. Similar to the claim in [4], we have the following claim.
Claim $1^{[4]}$. For any vertex $u \in V(G)$, it holds that $\sum_{i=1}^{3}\left[d_{i}(u)(\Delta(G)+4-d(u)-i)\right] \leq d(u)-1$.

By Claim 1 , for any $u \in V(G)$ with $d(u) \geq 4$, we have the following claim.

Claim 2. (1) There is no $4^{-}$-vertex adjacent to any $3^{-}$vertex.

(2) If $u$ is a 5 -vertex of $G$, then $d_{1}(u)=0$ and $d_{3^{-}}(u)=0$.

(3) If $u$ is a 6 -vertex of $G$, then $d_{2^{-}}(u) \leq 1$ and if $d_{2}(u)=1$, then $d_{3}(u) \leq 1$.

(4) If $u$ is a $l$-vertex of $G$ with $l \geq 7$, then $d_{1}(u) \leq\left\lfloor\frac{l-1}{3}\right\rfloor$.

Let $H$ be the graph obtained by removing all leaves of $G$. By claim2, $H$ is a connected planar graph with $\Delta(H) \geq 2$, and we have the following claim.

Claim 3. Let $v$ be a vertex of $H$, if $d_{H}(v)=2$ or $d_{H}(v)=3$, then the neighbors of $v$ must be $5^{+}$-vertices in $H$.

In order to complete the proof, we use the discharging method. Using Euler's formula

$$
V(H)|-| E(H)|+| F(H) \mid=2,
$$

then $\sum_{v \in V(H)}\left(d_{H}(v)-4\right)+\sum_{f \in F(H)}\left(d_{H}(f)-4\right)=-8$.

First, we give an initial charge function $w(v)=d_{H}(v)-4$ for every $\quad v \in V(G) \quad$ and $w(f)=d_{H}(f)-4$ for every $f \in F(H)$. Next, we design a discharging rule and redistribute weights accordingly. Let $w^{\prime}$ be the new charge after the discharging. We will show that $w^{\prime}(x) \geq 0$ for all $x \in V(H) \cup F(H)$. This leads to the following contradiction:

$$
0 \leq \sum_{x \in V(H) \cup F(H)} w^{\prime}(x)=\sum_{x \in V(H) \cup F(H)} w(x)=-8<0 .
$$

Hence, this demonstrates that no such a counterexample can exist. The discharging rule is defined as follow:

(R) For each $5^{+}$-vertices $u$ of $H$, gives 1 to each adjacent 2-vertex and gives $\frac{1}{3}$ to each adjacent 3 -vertex.

By rule (R), we have the following results:

1. For each 5-vertex $u \in V(G)$, by claim 2, $d_{H}(u)=5$ and $u$ has at most one neighbor of $3^{-}$-vertex in $H$. So $w^{\prime}(u) \geq 5-4-1=0$. 
2. For each 6-vertex $u \in V(G)$, by claim 2, $d_{2^{-}}(u) \leq 1$. And we have

(1) if $d_{2^{-}}(u)=0$, then $d_{H}(u)=6$, and the neighbors of $u$ must be all $3^{+}$-vertex in $H$, So $w^{\prime}(u) \geq 6-4-\frac{6}{3}=0$.

(2) if $d_{2^{-}}(u)=1$, then $d_{3}(u) \leq 1$, So $w^{\prime}(u)=6-4-d_{2^{-}}(u)-\frac{d_{3}(u)}{3} \geq 2-1-\frac{1}{3}>0$.

3. For each $l$-vertex $u \in V(G)$ with $l \geq 7$, by claim 1 , we have

$$
\begin{aligned}
& l-1-(\Delta-l+3) d_{1}(u)-(\Delta-l+2) d_{2}(u) \\
& -(\Delta-l+1) d_{3}(u) \geq 0 .
\end{aligned}
$$

So (1) if $d_{2^{-}}(u)=0$, then

$$
\begin{aligned}
& w^{\prime}(u)=l-4-d_{2^{-}}(u)-\frac{d_{3}(u)}{3} \geq l-4-\frac{l}{3}>0 . \\
& \text { (2) if } d_{2^{-}}(u)=1 \text {, then }
\end{aligned}
$$

$$
\begin{aligned}
w^{\prime}(u) & =l-4-d_{2^{-}}(u)-\frac{d_{3}(u)}{3} . \\
& \geq l-4-1-\frac{l-1}{3} \geq 0
\end{aligned}
$$

(3) if $d_{2^{-}}(u)=2$, by claim 1 , we have

$$
3 d_{1}(u)+2 d_{2}(u)+d_{3}(u) \leq l-1,
$$

which induces that $d_{3}(u) \leq l-5$. Then

$$
\begin{aligned}
w^{\prime}(u) & =l-4-d_{2^{-}}(u)-\frac{d_{3}(u)}{3} . \\
& \geq 1-4-2-\frac{l-5}{3}>0
\end{aligned}
$$

(4) if $d_{2^{-}}(u) \geq 3$, then

$$
\begin{aligned}
w^{\prime}(u) & =l-4-d_{1}(u)-d_{2}(u)-\frac{d_{3}(u)}{3} \\
& =\left[l-1-(\Delta-l+3) d_{1}(u)\right.
\end{aligned}
$$

$$
\begin{aligned}
& \left.-(\Delta-l+2) d_{2}(u)-(\Delta-l+1) d_{3}(u)\right] \\
& +(\Delta-l+2) d_{1}(u)+(\Delta-l+1) d_{2}(u) \\
& +\left(\Delta-l+\frac{2}{3}\right) d_{3}(u)-3 \\
& \geq 2 d_{1}(u)+d_{2}(u)+\frac{2 d_{3}(u)}{3} \geq 0 .
\end{aligned}
$$

4. For each 2-vertex or 3-vertex $u$ in $H$, by claim 3, we have $\quad w^{\prime}(u) \geq 2-4+2=0$ or $w^{\prime}(u) \geq 3-4+3 \cdot \frac{1}{3}=0$.

5. For each face $f$ in $H$, since $H$ is also a planar graph with girth at least 4 , then $d_{H}(f) \geq 4$, and we have $w^{\prime}(f)=w(f) \geq 0$.

From above discussion, we have $\sum_{x \in V(H) \cup F(H)} w^{\prime}(x) \geq 0$. It is a contradiction, which completes the proof of Theorem 1.1.

\section{ACKNOWLEDGMENT}

This work was supported by the Natural Science Foundation of Shandong Provence (ZR2016AM01) and the Scientific Research Foundation of University of Jinan (XKY1612)

\section{REFERENCES}

[1] B. Seamone, The 1-2-3 conjecture and related problems: a survey, arXiv:1211.5122.

[2] D. Huang and W. Wang, Adjacent vertex distinguishing total coloring of planar graphs with large maximum degree, (in Chinese), Sci. Sin. Math., 42(2)(2012), 151-164.

[3] J. A. Bondy and U. S. R. Murty, Graph Theory with Applications, North-Holland, New York, 1976.

[4] J. Wang, J. Cai and B. Qiu, Neighbor Sum Distinguishing Total Choosability of Planar Graphs without Adjacent Triangles, Theoretical Computer Science, (2016) DOI 10.1016/j.tcs.2016.11.003.

[5] M. Pilsniak and M. Wozniak, On the total-neighbor-distinguishing index by sums, Graph and Combin., 31(2015),771-782.

[6] W. Wang and D. Huang, The adjacent vertex distinguishing total coloring of planar graphs, J. Combin. Optim., 27(2014), 379-396.

[7] Y. Wang and W. Wang, Adjacent vertex distinguishing total coloring of outerplanar graphs, J. Combin. Optim., 19(2010), 123-133.

[8] Z. Zhang, X. Chen, J. Li, B.Yao, X. Lu and J.Wang, On adjacent-vertexdistinguishing total coloring of graphs, Sci. China Ser. A Mathematics, 48(3)(2005), 289-299. 